\title{
Egg-eating predators in interaction with age-structured prey population
}

\author{
Ruslan Andrusyak ${ }^{1}$, Ivanna Andrusyak ${ }^{2}$, and Ulyana Telyuk ${ }^{3}$ \\ ${ }^{1}$ Department of Mechanics and Mathematics, Ivan Franko National University of Lviv, Ukraine \\ ${ }^{2}$ Institute of Applied Mathematics and Fundamental Sciences, Lviv Polytechnic National University, Ukraine \\ ${ }^{3}$ Department of Mechanics and Mathematics, Ivan Franko National University of Lviv, Ukraine
}

Received: 9 November 2016, Accepted: 5 March 2017

Published online: 25 August 2017.

\begin{abstract}
We investigate a predator-prey model for egg-eating predators in which the prey population is assumed to have an age structure. By the method of characteristics, this model reduces to a system of integral equations. Then a generalization of the Banach fixed-point theorem is used to show, under relatively mild conditions, the existence of a unique, global, weak solution to the population problem. Furthermore, this methodology allows us to generate a sequence of iterates, called the Picard iterates, that converges to the solution. Also, we strengthen the assumptions of the existence-uniqueness theorem to establish the validity of the corresponding conservation law in integral form. Thus we prove a result which shows the coexistence of both predator and prey species over a long time.
\end{abstract}

Keywords: Predator-prey model, age structure, the method of characteristics, contraction mapping, the Picard iteration.

\section{Introduction}

Population dynamics has traditionally been the dominant branch of mathematical biology. Population models play a critical role in helping us to understand the dynamic processes involved, in making practical predictions, and thus in better understanding the natural world.

The construction and investigation of models for the population dynamics of predator-prey interactions have remained an important area in theoretical ecology since the famous Lotka-Volterra equations. The mathematics used to study such models often takes into account the structure of populations, provided that this structure influences the size of each species in a major way. The application of physiologically structured models to describe the behavior of biological systems has attracted the interest of many researchers and has a long standing tradition. The books by Charlesworth [1], Metz and Diekmann [2], Cushing [3], and Murray [4] give a good survey as well as the wide spectrum of applicability of such models.

Allowing for an age structure, predator-prey relationships are usually governed by partial differential equations or mixed PDE-ODE systems, possibly with constant or distributed time delay. Sometimes predator-prey models are considered in which only the predator population has an age structure that significantly affects its fecundity. In this case the age structure of the prey population is insignificant in comparison to that of the predators (see Cushing and Saleem [5]). Other researchers study predator-prey interactions with predation dependent on age of prey. The dynamics of the predator and prey populations are shown to depend substantially on what ages of prey are eaten by predators. In particular, two cases are studied: where the predators eat all ages of prey indiscriminately, and where the predators eat only eggs (or newborns, equivalently). For example, in Gurtin and Levine [6], the limiting dynamics of such 
predator-prey interactions are considered. Indiscriminate eating is found to lead to stable periodic oscillations in numbers of predator and prey, such as occur in the Lotka-Volterra equations, while egg eating leads to oscillations which increase rapidly in amplitude and result, ultimately, in the extinction of both predator and prey. Other results for corresponding predator-prey models also concern the existence and stability properties of nonnegative equilibria solutions (see, e.g., [7, $8,9,10])$.

Our research is devoted to a predator-prey model for egg-eating predators in which the prey population is assumed to have an age structure. The goal is to obtain sufficient conditions for the existence of a unique, global, weak solution to the relevant mathematical problem; this solution determines how the age structure of the prey population and the total predator population evolve in time. We study the simple case when the prey dynamics is modeled by the McKendrick-von Foerster equation. Such an equation is hyperbolic, and the fundamental idea associated with hyperbolic equations is the notion of a characteristic, a curve in space-time along which signals propagate. So, using the method of characteristics, which is highly effective for investigating hyperbolic continuous-time models (see, e.g., Logan [11], Brauer and Castillo-Chavez [12]), the solution of the population problem can be expressed as a fixed point of some appropriately chosen integral operator in a suitable metric space. Then a generalization of the Banach fixed-point theorem is used to show, under relatively mild conditions, the existence of a unique, global solution to the problem. Furthermore, this methodology allows us to generate a sequence of iterates, called the Picard iterates, that converges to the solution. Also, we strengthen the assumptions of the existence-uniqueness theorem to establish the validity of the corresponding conservation law in integral form. Note that, under our assumptions, the predator and prey populations will never vanish as time increases. Thus we prove a result which shows the coexistence of both predator and prey species over a long time.

\section{Population model with age distribution}

Let $u(x, t)$ be an unknown density of a population at time $t$ with respect to an age variable $x$, so that the population at time $t$ between ages $x_{1}$ and $x_{2}$ is $\int_{x_{1}}^{x_{2}} u(x, t) d x$. Therefore, the total number of individuals at any time $t$ is $\int_{0}^{L} u(x, t) d x$, where $L$ is the maximum lifetime.

We assume that members leave the population through death, and that there is an age-dependent death rate $m(x)$. This means that over the time interval from $t_{1}$ to $t_{2}$ the number $\int_{t_{1}}^{t_{2}} \int_{x_{1}}^{x_{2}} m(x) u(x, t) d x$ of individuals with ages between $x_{1}$ and $x_{2}$ die. Thus we obtain the conservation law in integral form

$$
\int_{x_{1}}^{x_{2}} u\left(x, t_{2}\right) d x-\int_{x_{1}}^{x_{2}} u\left(x, t_{1}\right) d x=\int_{t_{1}}^{t_{2}} u\left(x_{1}, t\right) d t-\int_{t_{1}}^{t_{2}} u\left(x_{2}, t\right) d t-\int_{t_{1}}^{t_{2}} \int_{x_{1}}^{x_{2}} m(x) u(x, t) d x d t
$$

for all $\left(x_{1}, t_{1}\right),\left(x_{2}, t_{2}\right) \in \Omega=\{(x, t): 0 \leq x \leq L, 0 \leq t<+\infty\}$.

Assuming smoothness of $u$, as well as continuity of $m$, equation (1) may be transformed into the single PDE

$$
\frac{\partial}{\partial t} u(x, t)+\frac{\partial}{\partial x} u(x, t)=-m(x) u(x, t) \quad \text { for } \quad(x, t) \in \Omega \text {. }
$$

Thus we obtained the McKendrick equation (1926), which is also known as the von Foerster equation (1959), because the same equation arises in cellular biology.

Next, we assume that the birth process is governed by a function $b(x, t)$ called the birth rate. Thus the total number of births (eggs) between time $t_{1}$ and time $t_{2}$ is $\int_{t_{1}}^{t_{2}} \int_{0}^{L} b(x, t) u(x, t) d x d t$. Since this quantity must also be $\int_{t_{1}}^{t_{2}} u(0, t) d t$, we obtain the renewal condition

$$
u(0, t)=\int_{0}^{L} b(x, t) u(x, t) d x, \quad \text { for } \quad t \geq 0 .
$$


In order to complete the model, we must specify an initial age distribution

$$
u(x, 0)=u_{0}(x), \quad \text { for } \quad 0 \leq x \leq L .
$$

Then the full model consists of the PDE (2) and the two auxiliary conditions (3), (4) (see [4], [11], [12]).

It is easy to see that the characteristic curves for equation (2) are the straight lines $x-t=$ const. Characteristics are the fundamental concept in the analysis of hyperbolic problems because PDEs simplify to ODEs along these curves. Thus differentiating the solution $u$ along the characteristics yields

$$
\frac{d u(\tau+x-t, \tau)}{d \tau}=-m(\tau+x-t) u(\tau+x-t, \tau) .
$$

This equation can be solved by separation of variables to get

$$
u(\tau+x-t, \tau)=u\left(\tau_{0}+x-t, \tau_{0}\right) \exp \left(-\int_{\tau_{0}}^{\tau} m\left(\tau_{1}+x-t\right) d \tau_{1}\right) .
$$

Make the change of variables $\xi=\tau_{1}+x-t$ to obtain

$$
u(\tau+x-t, \tau)=u\left(\tau_{0}+x-t, \tau_{0}\right) \exp \left(-\int_{\tau_{0}+x-t}^{\tau+x-t} m(\xi) d \xi\right) .
$$

Denote $\pi(x):=\exp \left(-\int_{0}^{x} m(\xi) d \xi\right)$, which is the probability of survival from birth to age $x$. Then $\pi(x) \pi\left(x_{0}\right)^{-1}=\exp \left(-\int_{x_{0}}^{x} m(\xi) d \xi\right)$, for any $x_{0}<x$, is the probability that an individual of age $x_{0}$ will survive to age $x$. We now assume that $\int_{0}^{L} m(x) d x=+\infty$, while the function $x \mapsto m(x)$ is locally integrable in $[0, L)$. Thus $\pi(L)=0$ (the probability to survive to the maximum possible age $L$ equals zero), and in the sequel, for simplicity of our investigation, we put (by definition) $\pi(x)=0$, and therefore $u(x, t)=0$, for all $x \geq L$. Moreover, the product of $\pi$ and any other function is interpreted to be zero whenever $x \geq L$ (even if the latter function is not defined on this interval).

Taking $\tau_{0}=0, \tau=t$, (i.e., considering the previous equation (6) on the characteristics that emanate from points $(x-t, 0)$ on the $x$ axis) and, similarly, taking $\tau_{0}=t-x, \tau=t$, (i.e., considering equation (6) on the characteristics that emanate from points $(0, t-x)$ on the $t$ axis) give

$$
\begin{aligned}
& u(x, t)=u(x-t, 0) \frac{\pi(x)}{\pi(x-t)}, \quad \text { for } \quad 0 \leq t \leq x, \\
& u(x, t)=u(0, t-x) \pi(x), \quad \text { for } \quad 0 \leq x \leq t .
\end{aligned}
$$

Definition 1. By a weak solution to the initial-boundary value problem (2), (3), (4) we mean a function $(x, t) \mapsto u(x, t)$ continuous in $[0, L] \times[0,+\infty)$ and satisfying the functional equations (7), along with conditions (3), (4).

We can easily show that if a weak solution to the initial-boundary value problem (2), (3), (4) is continuously differentiable, then this solution is classical, that is, it makes sense to calculate its first derivatives and substitute them into equation (2).

\section{Predator-prey model for egg-eating predators}

We consider a population of prey with age density $u(x, t)$ at time $t$ with respect to an age variable $x$. Assume that, in the absence of predators, the prey population is modeled by the initial-boundary value problem (2), (3), (4). Now let us introduce a predator population that consumes the eggs of the prey population. The total number of predators at any time $t$ is $P(t)$, and we do not consider age structure in this population. It is interesting that egg-eating predators is one 
of the theories posed for the extinction of the dinosaurs. Since predators eat only eggs, the PDE (2) is unaffected. What is affected is the number of offspring (eggs) produced. Thus we no longer have the renewal condition (3), but we must include a predation term that decreases the number of eggs. The simplest model is the Lotka-Volterra model, which requires that the number of eggs eaten be proportional to the product of the number of eggs and the number of predators. Therefore, taking into account that the function $u(0, t)$ must be nonnegative, we have

$$
u(0, t)=\left((1-\varkappa P(t)) \int_{0}^{L} b(x, t) u(x, t) d x\right)^{+} \quad \text { for } \quad t \geq 0,
$$

where $\varkappa$ is the predation rate, and $(x)^{+}:=\max \{x, 0\}$ for any real $x$.

Finally, we impose the Lotka-Volterra dynamics on the predator population given by

$$
\frac{d P(t)}{d t}=-\mu P(t)+\beta P(t) \int_{0}^{L} b(x, t) u(x, t) d x \text { for } t \geq 0,
$$

where $\mu$ is the per capita mortality rate. Hence, in the absence of eggs, predators die out. Initially, we take

$$
P(0)=P_{0}
$$

In summary, the predator-prey model is given by the mixed PDE-ODE system (2), (9), the boundary condition (8), and the initial conditions (4), (10) (see [11]).

We remark that if the predators consumed prey other than eggs, then a predation term would have to be included on the right side of the dynamical equation (2). Note also that the ODE (9) is equivalent to the integral equation

$$
P(t)=P(0) \exp (-\mu t)+\beta \int_{0}^{t} P(\tau) \exp (-\mu(t-\tau))\left(\int_{0}^{L} b(x, \tau) u(x, \tau) d x\right) d \tau \quad \text { for } \quad t \geq 0 .
$$

Definition 2. By a weak solution of the predator-prey model we mean a pair of functions $(u, P)$, where $(x, t) \mapsto u(x, t)$ and $t \mapsto P(t)$ are continuous in their respective domains $[0, L] \times[0,+\infty)$ and $[0,+\infty)$, such that equations (7), (11), along with conditions (4), (8), (10), are satisfied.

\section{Reduction of the predator-prey model to a system of integral equations}

Substitute conditions (4), (8), (10) into the corresponding equations (7), (11) to obtain

$$
\begin{aligned}
& u(x, t)=u_{0}(x-t) \frac{\pi(x)}{\pi(x-t)}, \quad \text { for } \quad 0 \leq t \leq x, \\
& u(x, t)=\left((1-\varkappa P(t-x)) \int_{0}^{L} b(\xi, t-x) u(\xi, t-x) d \xi\right)^{+} \pi(x), \quad \text { for } \quad 0 \leq x \leq t, \\
& P(t)=P_{0} \exp (-\mu t)+\beta \int_{0}^{t} P(\tau) \exp (-\mu(t-\tau))\left(\int_{0}^{L} b(x, \tau) u(x, \tau) d x\right) d \tau, \quad \text { for } t \geq 0 .
\end{aligned}
$$

It is easy to show that a solution of system (12) also satisfies conditions (4), (8), (10). Then we have the following proposition.

Proposition 1. Finding a solution of the predator-prey model is equivalent to solving the system of integral equations (12) for the unknown functions $u:[0, L] \times[0,+\infty) \rightarrow \mathbb{R}$ and $P:[0,+\infty) \rightarrow \mathbb{R}$. 
Let us denote by $B(t)$ the number of births (eggs) in unit time after subtracting the number of eggs eaten by predators, that is $B(t):=u(0, t)$. Then, using this notation, rewrite system (12) in the equivalent form

$$
\begin{aligned}
& u(x, t)=u_{0}(x-t) \frac{\pi(x)}{\pi(x-t)}, \quad \text { for } \quad 0 \leq t \leq x, \\
& u(x, t)=B(t-x) \pi(x), \quad \text { for } \quad 0 \leq x \leq t, \\
& B(t)=\left((1-\varkappa P(t)) \int_{0}^{L} b(x, t) u(x, t) d x\right)^{+}, \quad \text { for } t \geq 0, \\
& P(t)=P_{0} \exp (-\mu t)+\beta \int_{0}^{t} P(\tau) \exp (-\mu(t-\tau))\left(\int_{0}^{L} b(x, \tau) u(x, \tau) d x\right) d \tau, \text { for } t \geq 0 .
\end{aligned}
$$

Using the first two relations in (13) and recalling our convention that $\pi(x)=0$, and therefore $u(x, t)=0$, for all $x \geq L$, the integral $\int_{0}^{L} b(x, t) u(x, t) d x$ can be transformed as follows:

$$
\begin{aligned}
& \int_{0}^{+\infty} b(x, t) u(x, t) d x=\int_{0}^{t} b(x, t) u(x, t) d x+\int_{t}^{+\infty} b(x, t) u(x, t) d x \\
& =\int_{0}^{t} b(x, t) B(t-x) \pi(x) d x+\int_{t}^{+\infty} b(x, t) u_{0}(x-t) \frac{\pi(x)}{\pi(x-t)} d x .
\end{aligned}
$$

Denoting $q(t):=\int_{t}^{+\infty} b(x, t) u_{0}(x-t) \frac{\pi(x)}{\pi(x-t)} d x$, rewrite system (13) in the equivalent form

$$
\begin{aligned}
& u(x, t)=u_{0}(x-t) \frac{\pi(x)}{\pi(x-t)}, \quad \text { for } \quad 0 \leq t \leq x, \\
& u(x, t)=B(t-x) \pi(x), \quad \text { for } \quad 0 \leq x \leq t \\
& B(t)=\left((1-\varkappa P(t))\left(\int_{0}^{t} b(x, t) B(t-x) \pi(x) d x+q(t)\right)\right)^{+}, \quad \text { for } \quad t \geq 0, \\
& P(t)=P_{0} \exp (-\mu t)+\beta \int_{0}^{t} P(\tau) \exp (-\mu(t-\tau))\left(\int_{0}^{\tau} b(x, \tau) B(\tau-x) \pi(x) d x+q(\tau)\right) d \tau, \quad \text { for } \quad t \geq 0 .
\end{aligned}
$$

Clearly, the first two relations decouple from the system, and we can consider just the last two integral equations for the total predator and egg populations $P(t)$ and $B(t)$

$$
\begin{aligned}
& B(t)=\left((1-\varkappa P(t))\left(\int_{0}^{t} b(x, t) B(t-x) \pi(x) d x+q(t)\right)\right)^{+}, \quad \text { for } \quad t \geq 0, \\
& P(t)=P_{0} \exp (-\mu t)+\beta \int_{0}^{t} P(\tau) \exp (-\mu(t-\tau))\left(\int_{0}^{\tau} b(x, \tau) B(\tau-x) \pi(x) d x+q(\tau)\right) d \tau, \quad \text { for } \quad t \geq 0 .
\end{aligned}
$$

Proposition 2. Suppose the compatibility condition

$$
u_{0}(0)=\left(\left(1-\varkappa P_{0}\right) \int_{0}^{L} b(x, 0) u_{0}(x) d x\right)^{+}
$$

holds; then finding a solution of the predator-prey model can be reduced to solving the system of integral equations (14) for the unknown functions B and P. More precisely,

- a weak solution $(u, P)$ of the predator-prey model gives the continuous solution $(B, P)$ of system (14), where the function $B=B(t)$ is explicitly determined by the formula $B(t)=\left((1-\varkappa P(t)) \int_{0}^{L} b(x, t) u(x, t) d x\right)^{+}$for $t \geq 0$ provided the continuity of $b$ (this condition on $b$ can be slightly weakened); 
- conversely, a continuous solution $(B, P)$ of system (14) explicitly determines the function $u=u(x, t)$ from the relations

$$
\begin{aligned}
& u(x, t)=u_{0}(x-t) \frac{\pi(x)}{\pi(x-t)}, \quad \text { for } \quad 0 \leq t \leq x, \\
& u(x, t)=B(t-x) \pi(x), \quad \text { for } \quad 0 \leq x \leq t
\end{aligned}
$$

and thus we have the weak solution $(u, P)$ of the predator-prey model, provided that the functions $u_{0}, \pi$ are continuous, and condition (15) is satisfied.

The proof of proposition 2 is trivial, so we only remark that the compatibility condition (15) is necessary to avoid a discontinuity in the function $u=u(x, t)$ along the line $x=t$.

\section{Existence-uniqueness theorem}

We formulate an existence-uniqueness theorem for the predator-prey model.

Theorem 1.Suppose the following conditions hold.

(1) $\varkappa, \mu, \beta$, and $P_{0}$ are nonnegative constants;

(2) the function $x \mapsto m(x)$ is nonnegative, locally Lebesgue integrable in $[0, L)$ such that $\int_{0}^{L} m(x) d x=+\infty($ then the function $x \mapsto \pi(x)$, defined by $\pi(x)=\exp \left(-\int_{0}^{x} m(\xi) d \xi\right)$ for $0 \leq x<L$ and $\pi(x)=0$ for $L \leq x<+\infty$, is continuous in $[0,+\infty))$;

(3) the function $x \mapsto u_{0}(x)$ is nonnegative, continuous in $[0, L]$;

(4) the function $(x, t) \mapsto b(x, t)$ is nonnegative, continuous in $t$ for each fixed $x$ and locally essentially bounded, measurable in $x$ for each fixed $t$ on $\Omega$ (meaning that on every compact subset of $\Omega$ there exists an essential upper bound for $b$ independent of $t$ );

(5) the compatibility condition (15) holds.

Then there exists a unique, nonnegative, global, weak solution $(u, P)$ of the predator-prey model.

Proof. The right sides of equations (14) can be regarded as a mapping $\mathscr{M}$ on the set of continuous vector functions $(B, P)$. That is, with each pair $(B, P)$ of functions continuous in the interval $[0, T](T>0)$ there is associated another pair of functions $\mathscr{M}(B, P)=\left(\mathscr{M}_{B}(B, P), \mathscr{M}_{P}(B, P)\right)$ defined, at each $0 \leq t \leq T$, by

$$
\begin{aligned}
& \mathscr{M}_{B}(B, P)(t)=\left((1-\varkappa P(t))\left(\int_{0}^{t} b(x, t) B(t-x) \pi(x) d x+q(t)\right)\right)^{+} \\
& \mathscr{M}_{P}(B, P)(t)=P_{0} \exp (-\mu t)+\beta \int_{0}^{t} P(\tau) \exp (-\mu(t-\tau))\left(\int_{0}^{\tau} b(x, \tau) B(\tau-x) \pi(x) d x+q(\tau)\right) d \tau .
\end{aligned}
$$

Therefore, system (14) may be written in the form $(B, P)=\mathscr{M}(B, P)$. Thus solving the predator-prey model is reduced to finding a fixed point of the mapping $(B, P) \mapsto \mathscr{M}(B, P)$.

Consider the Banach space $C\left([0, T] ; \mathbb{R}^{2}\right)$ consisting of all continuous two-dimensional vector functions on $[0, T]$, equipped with the uniform norm. Let $\mathscr{B}(T)$ be the closed subset of the space $C\left([0, T] ; \mathbb{R}^{2}\right)$ that consists of all vector functions $(B, P)$ such that

- the function $B$ satisfies the inequality $0 \leq B(t) \leq k_{2} \exp \left(k_{1} t\right)$ for all $0 \leq t \leq T$, where $k_{1}:=\sup _{t \in[0, T]} \operatorname{ess~sup}_{x \in[0, L]} b(x, t)$ (recall that, by assumption, there exists an essential upper bound for $b$ independent of $t$ ) and $k_{2}:=k_{1} \int_{0}^{L} u_{0}(x) d x$;

- the function $P$ satisfies the inequality $0 \leq P(t) \leq P_{0} \exp \left(\beta k_{2} k_{1}^{-1}\left(\exp \left(k_{1} t\right)-1\right)\right)$ for all $0 \leq t \leq T$. 
We will treat the subset $\mathscr{B}(T)$ as a metric space using the metric induced by the uniform norm, that is, defining the distance between any vector functions $\left(B^{1}, P^{1}\right)$ and $\left(B^{2}, P^{2}\right)$ by

$$
\left\|\left(B^{1}, P^{1}\right)-\left(B^{2}, P^{2}\right)\right\|_{\infty, T}:=\max _{t \in[0, T]}\left|B^{1}(t)-B^{2}(t)\right|+\max _{t \in[0, T]}\left|P^{1}(t)-P^{2}(t)\right| .
$$

Note that every closed subset of a complete metric space is complete.

Thus, to prove the existence-uniqueness result for the predator-prey model, let us show that the mapping $\mathscr{M}$ has precisely one fixed point in the metric space $\mathscr{B}(T)$. To this end, by a generalization of the Banach fixed-point theorem, we must show that this space is invariant under the mapping $\mathscr{M}$, and some its iterate $\mathscr{M}^{n}$ is a contraction on the space $\mathscr{B}(T)$ with respect to the corresponding norm.

It is easily seen that the functions $\mathscr{M}_{B}(B, P)$ and $\mathscr{M}_{P}(B, P)$ are nonnegative, continuous in $[0, T]$ if so are the functions $B$ and $P$; that is, $\mathscr{M}(B, P) \in C\left([0, T] ; \mathbb{R}_{+}^{2}\right)$ whenever $(B, P) \in C\left([0, T] ; \mathbb{R}_{+}^{2}\right)$. Further, for any pair $(B, P)$ in $\mathscr{B}(T)$, we obtain the estimates

$$
\begin{aligned}
& \mathscr{M}_{B}(B, P)(t) \leq \int_{0}^{t} b(x, t) B(t-x) \pi(x) d x+q(t) \leq k_{1} \int_{0}^{t} B(t-x) d x+k_{2} \\
& \leq k_{1} k_{2} \int_{0}^{t} \exp \left(k_{1}(t-x)\right) d x+k_{2}=k_{2}\left(\exp \left(k_{1} t\right)-1\right)+k_{2}=k_{2} \exp \left(k_{1} t\right) \quad \text { for } \quad 0 \leq t \leq T, \\
& \mathscr{M}_{P}(B, P)(t) \leq P_{0}+\beta \int_{0}^{t} P(\tau)\left(k_{1} \int_{0}^{\tau} B(\tau-x) d x+k_{2}\right) d \tau \\
& \leq P_{0}+\beta P_{0} \int_{0}^{t} \exp \left(\beta k_{2} k_{1}^{-1}\left(\exp \left(k_{1} \tau\right)-1\right)\right)\left(k_{1} k_{2} \int_{0}^{\tau} \exp \left(k_{1}(\tau-x)\right) d x+k_{2}\right) d \tau \\
& =P_{0}+\beta P_{0} \int_{0}^{t} \exp \left(\beta k_{2} k_{1}^{-1}\left(\exp \left(k_{1} \tau\right)-1\right)\right) k_{2} \exp \left(k_{1} \tau\right) d \tau=P_{0}+P_{0} \int_{0}^{\beta k_{2} k_{1}^{-1}\left(\exp \left(k_{1} t\right)-1\right)} \exp \tau_{1} d \tau_{1} \\
& =P_{0}+P_{0}\left(\exp \left(\beta k_{2} k_{1}^{-1}\left(\exp \left(k_{1} t\right)-1\right)\right)-1\right)=P_{0} \exp \left(\beta k_{2} k_{1}^{-1}\left(\exp \left(k_{1} t\right)-1\right)\right) \quad \text { for } \quad 0 \leq t \leq T .
\end{aligned}
$$

Thus, we have proved that the space $\mathscr{B}(T)$ is invariant under the mapping $\mathscr{M}$, that is, $\mathscr{M}(B, P) \in \mathscr{B}(T)$ whenever $(B, P) \in \mathscr{B}(T)$. Now let us show that there is a positive integer $n$ such that $\mathscr{M}^{n}$ ( $n$-th iterate of $\mathscr{M}$ ) is a contraction mapping. Taking $\left(B^{1}, P^{1}\right),\left(B^{2}, P^{2}\right)$ to be in the space $\mathscr{B}(T)$, we obtain the following estimates:

$$
\begin{aligned}
& \left|\mathscr{M}_{B}\left(B^{1}, P^{1}\right)(t)-\mathscr{M}_{B}\left(B^{2}, P^{2}\right)(t)\right| \leq\left(1+\varkappa P^{1}(t)\right) \int_{0}^{t} b(x, t)\left|B^{1}(t-x)-B^{2}(t-x)\right| \pi(x) d x \\
& +\varkappa\left|P^{1}(t)-P^{2}(t)\right|\left(\int_{0}^{t} b(x, t) B^{2}(t-x) \pi(x) d x+q(t)\right) \\
& \leq\left(1+\varkappa P^{1}(t)\right) k_{1} \int_{0}^{t}\left|B^{1}(t-x)-B^{2}(t-x)\right| d x+\varkappa\left|P^{1}(t)-P^{2}(t)\right|\left(k_{1} \int_{0}^{t} B^{2}(t-x) d x+k_{2}\right) \\
& \leq\left(1+\varkappa P_{0} \exp \left(\beta k_{2} k_{1}^{-1}\left(\exp \left(k_{1} t\right)-1\right)\right)\right) k_{1} \int_{0}^{t}\left|B^{1}(t-x)-B^{2}(t-x)\right| d x+\varkappa k_{2} \exp \left(k_{1} t\right)\left|P^{1}(t)-P^{2}(t)\right| \\
& \leq C_{1} \int_{0}^{t}\left|B^{1}(\tau)-B^{2}(\tau)\right| d \tau+C_{2}\left|P^{1}(t)-P^{2}(t)\right| \text { for } 0 \leq t \leq T,
\end{aligned}
$$


where $C_{1}:=\left(1+\varkappa P_{0} \exp \left(\beta k_{2} k_{1}^{-1}\left(\exp \left(k_{1} T\right)-1\right)\right)\right) k_{1}, C_{2}:=\varkappa k_{2} \exp \left(k_{1} T\right) ;$ and also

$$
\begin{aligned}
& \left|\mathscr{M}_{P}\left(B^{1}, P^{1}\right)(t)-\mathscr{M}_{P}\left(B^{2}, P^{2}\right)(t)\right| \leq \beta \int_{0}^{t} P^{1}(\tau) \exp (-\mu(t-\tau))\left(\int_{0}^{\tau} b(x, \tau)\left|B^{1}(\tau-x)-B^{2}(\tau-x)\right| \pi(x) d x\right) d \tau \\
& +\beta \int_{0}^{t}\left|P^{1}(\tau)-P^{2}(\tau)\right| \exp (-\mu(t-\tau))\left(\int_{0}^{\tau} b(x, \tau) B^{2}(\tau-x) \pi(x) d x+q(\tau)\right) d \tau \\
& \leq \beta \int_{0}^{t} P^{1}(\tau)\left(k_{1} \int_{0}^{\tau}\left|B^{1}(\tau-x)-B^{2}(\tau-x)\right| d x\right) d \tau+\beta \int_{0}^{t}\left|P^{1}(\tau)-P^{2}(\tau)\right|\left(k_{1} \int_{0}^{\tau} B^{2}(\tau-x) d x+k_{2}\right) d \tau \\
& \leq \beta k_{1} \int_{0}^{t} P_{0} \exp \left(\beta k_{2} k_{1}^{-1}\left(\exp \left(k_{1} \tau\right)-1\right)\right)\left(\int_{0}^{\tau}\left|B^{1}(\tau-x)-B^{2}(\tau-x)\right| d x\right) d \tau+\beta k_{2} \int_{0}^{t} \exp \left(k_{1} \tau\right)\left|P^{1}(\tau)-P^{2}(\tau)\right| d \tau \\
& \leq C_{3} \int_{0}^{t}\left|B^{1}(\tau)-B^{2}(\tau)\right| d \tau+C_{4} \int_{0}^{t}\left|P^{1}(\tau)-P^{2}(\tau)\right| d \tau \text { for } \quad 0 \leq t \leq T,
\end{aligned}
$$

where $C_{3}:=\beta k_{1} P_{0} \int_{0}^{T} \exp \left(\beta k_{2} k_{1}^{-1}\left(\exp \left(k_{1} \tau\right)-1\right)\right) d \tau, C_{4}:=\beta k_{2} \exp \left(k_{1} T\right)$.

From the last estimates it follows that

$$
\begin{aligned}
& \left|\mathscr{M}_{B}^{2}\left(B^{1}, P^{1}\right)(t)-\mathscr{M}_{B}^{2}\left(B^{2}, P^{2}\right)(t)\right| \leq C_{1} \int_{0}^{t}\left|\mathscr{M}_{B}\left(B^{1}, P^{1}\right)(\tau)-\mathscr{M}_{B}\left(B^{2}, P^{2}\right)(\tau)\right| d \tau \\
& +C_{2}\left|\mathscr{M}_{P}\left(B^{1}, P^{1}\right)(t)-\mathscr{M}_{P}\left(B^{2}, P^{2}\right)(t)\right| \leq C_{1} \int_{0}^{t}\left(C_{1} \int_{0}^{\tau}\left|B^{1}\left(\tau_{1}\right)-B^{2}\left(\tau_{1}\right)\right| d \tau_{1}+C_{2}\left|P^{1}(\tau)-P^{2}(\tau)\right|\right) d \tau \\
& +C_{2}\left(C_{3} \int_{0}^{t}\left|B^{1}(\tau)-B^{2}(\tau)\right| d \tau+C_{4} \int_{0}^{t}\left|P^{1}(\tau)-P^{2}(\tau)\right| d \tau\right) \\
& \leq C_{5}\left(\int_{0}^{t}\left|B^{1}(\tau)-B^{2}(\tau)\right| d \tau+\int_{0}^{t}\left|P^{1}(\tau)-P^{2}(\tau)\right| d \tau\right) \quad \text { for } \quad 0 \leq t \leq T
\end{aligned}
$$

and, in the same way,

$$
\left|\mathscr{M}_{P}^{2}\left(B^{1}, P^{1}\right)(t)-\mathscr{M}_{P}^{2}\left(B^{2}, P^{2}\right)(t)\right| \leq C_{5}\left(\int_{0}^{t}\left|B^{1}(\tau)-B^{2}(\tau)\right| d \tau+\int_{0}^{t}\left|P^{1}(\tau)-P^{2}(\tau)\right| d \tau\right) \quad \text { for } \quad 0 \leq t \leq T,
$$

where the constant $C_{5}$ are easily determined. From the last two inequalities we derive the following one:

$$
\left\|\mathscr{M}^{2}\left(B^{1}, P^{1}\right)-\mathscr{M}^{2}\left(B^{2}, P^{2}\right)\right\|_{\infty, t} \leq C_{5} \int_{0}^{t}\left\|\left(B^{1}, P^{1}\right)-\left(B^{2}, P^{2}\right)\right\|_{\infty, \tau} d \tau \quad \text { for all } \quad 0 \leq t \leq T,
$$

and the constant $C_{5}$ does not depend on the choice of $\left(B^{1}, P^{1}\right)$ and $\left(B^{2}, P^{2}\right)$ in the space $\mathscr{B}(T)$.

Using this estimate, we obtain

$$
\begin{aligned}
& \left\|\mathscr{M}^{4}\left(B^{1}, P^{1}\right)-\mathscr{M}^{4}\left(B^{2}, P^{2}\right)\right\|_{\infty, t} \leq C_{5} \int_{0}^{t}\left\|\mathscr{M}^{2}\left(B^{1}, P^{1}\right)-\mathscr{M}^{2}\left(B^{2}, P^{2}\right)\right\|_{\infty, \tau} d \tau \\
& \leq C_{5}^{2} \int_{0}^{t} \int_{0}^{\tau}\left\|\left(B^{1}, P^{1}\right)-\left(B^{2}, P^{2}\right)\right\|_{\infty, \tau_{1}} d \tau_{1} d \tau \leq C_{5}^{2} \frac{t^{2}}{2}\left\|\left(B^{1}, P^{1}\right)-\left(B^{2}, P^{2}\right)\right\|_{\infty, t} ;
\end{aligned}
$$

and therefore,

$$
\begin{aligned}
& \left\|\mathscr{M}^{6}\left(B^{1}, P^{1}\right)-\mathscr{M}^{6}\left(B^{2}, P^{2}\right)\right\|_{\infty, t} \leq C_{5} \int_{0}^{t}\left\|\mathscr{M}^{4}\left(B^{1}, P^{1}\right)-\mathscr{M}^{4}\left(B^{2}, P^{2}\right)\right\|_{\infty, \tau} d \tau \\
& \leq C_{5}^{3} \int_{0}^{t} \frac{\tau^{2}}{2}\left\|\left(B^{1}, P^{1}\right)-\left(B^{2}, P^{2}\right)\right\|_{\infty, \tau} d \tau \leq C_{5}^{3} \frac{t^{3}}{6}\left\|\left(B^{1}, P^{1}\right)-\left(B^{2}, P^{2}\right)\right\|_{\infty, t} ;
\end{aligned}
$$


and so on.

Mathematical induction can be used to prove that the following estimate holds for all positive integers $n$ and all $t \in[0, T]$ :

$$
\left\|\mathscr{M}^{2 n}\left(B^{1}, P^{1}\right)-\mathscr{M}^{2 n}\left(B^{2}, P^{2}\right)\right\|_{\infty, t} \leq C_{5}^{n} \frac{t^{n}}{n !}\left\|\left(B^{1}, P^{1}\right)-\left(B^{2}, P^{2}\right)\right\|_{\infty, t}
$$

Thus, in general, the mapping $\mathscr{M}$ itself is not a contraction on the space $\mathscr{B}(T)$, but some its iterate $\mathscr{M}^{2 n}$ is a contraction, provided that $n$ is chosen sufficiently large to satisfy the inequality $\frac{\left(C_{5} T\right)^{n}}{n !}<1$.

Consequently, using a generalization of the Banach fixed-point theorem, the mapping $\mathscr{M}$ has precisely one fixed point in the metric space $\mathscr{B}(T)$ for all positive $T$. This fixed point is a solution of system (14) in the interval $[0, T]$. Taking into account that the value $T$ may be chosen arbitrarily large, we may extend this solution to any finite time $T_{1}$, where $T_{1}>T$, and therefore we obtain a global existence result, guaranteeing the existence of a unique solution $(B, P)$ of system (14) for all $t$ in $[0,+\infty)$, where $B$ and $P$ are nonnegative and continuous. Then applying proposition 2 gives the unique, nonnegative, global, weak solution $(u, P)$ of the predator-prey model. Thus the theorem is proved.

\section{Validity of the conservation law in integral form}

We remark that the PDE (2) arises from the conservation law in integral form (1), and the integral form of this law holds true even though the functions $u=u(x, t)$ and $m=m(x)$ may not meet the requirements of smoothness and continuity, respectively, imposed by the PDE. Now we strengthen the assumptions of the previous theorem to establish the validity of the conservation law (1) for a weak solution of the predator-prey model.

Theorem 2.In addition to the assumptions of theorem 1, suppose the following conditions hold:

(1) the function $x \mapsto \pi(x)$ is Lipschitz in $[0, L]$, or equivalently, $x \mapsto \pi^{\prime}(x)$ is essentially bounded in $(0, L)$ (recall that $\pi^{\prime}(x)=-\exp \left(-\int_{0}^{x} m(\xi) d \xi\right) m(x)$ for $\left.0 \leq x<L\right) ;$

(2) the function $x \mapsto u_{0}(x)$ is Lipschitz in $[0, L]$;

(3) the function $(x, t) \mapsto b(x, t)$ is locally Lipschitz in both variables on $\Omega$.

Then the weak solution, whose existence is guaranteed by theorem 1, also satisfies the integral form of the conservation law (1).

We remark that any function with continuous first derivatives is locally Lipschitz. Thus the property of being locally Lipschitz is stronger than continuity, yet weaker than continuous differentiability.

Proof. First show that, under the conditions stated in theorem 2, a weak solution of the predator-prey model is locally Lipschitz in $\Omega$. Indeed, making the change of variables, we rewrite relations (17) for the mapping $\mathscr{M}$ in the form

$$
\begin{aligned}
& \mathscr{M}_{B}(B, P)(t)=\left((1-\varkappa P(t))\left(\int_{0}^{t} b(t-\xi, t) B(\xi) \pi(t-\xi) d \xi+q(t)\right)\right)^{+} \\
& \mathscr{M}_{P}(B, P)(t)=P_{0} \exp (-\mu t)+\beta \int_{0}^{t} P(\tau) \exp (-\mu(t-\tau))\left(\int_{0}^{\tau} b(\tau-\xi, \tau) B(\xi) \pi(\tau-\xi) d \xi+q(\tau)\right) d \tau
\end{aligned}
$$

From these representations it is easily seen that the function $\mathscr{M}_{B}(B, P)$ is Lipschitz in $[0, T]$ if $B$ is continuous and $P$ is Lipschitz, but a sufficient condition for $\mathscr{M}_{P}(B, P)$ to be Lipschitz in $[0, T]$ is that the functions $B$ and $P$ be both continuous, provided that $\pi, u_{0}$, and $b$ satisfy Lipschitz conditions of theorem 2 . Therefore, if $B$ and $P$ are required to be both continuous, then the functions $\mathscr{M}_{B}(\mathscr{M}(B, P))$ and $\mathscr{M}_{P}(\mathscr{M}(B, P))$ are both Lipschitz in the interval $[0, T]$. It follows immediately that a fixed point of the mapping $(B, P) \mapsto \mathscr{M}(B, P)$ in $C\left([0, T] ; \mathbb{R}^{2}\right)$ satisfies a Lipschitz condition in $[0, T]$ for all $T>0$, and therefore the solution of system (14) defined for all $t$ in $[0,+\infty)$ is locally Lipschitz in this interval. 
Then, applying proposition 2, we deduce that a weak solution $(u, P)$ of the predator-prey model is locally Lipschitz in $\Omega$.

Further, prove the validity of the conservation law (1) for a weak solution of our model. Let $\Omega(T):=[0, L] \times[0, T]$. To prove this fact, it suffices to show that if Lipschitz functions $u: \Omega(T) \rightarrow \mathbb{R}$ and $P:[0, T] \rightarrow \mathbb{R}$ satisfy equations (12), and therefore $u$ satisfies equations (7), then the integral equation (1) holds for all $\left(x_{1}, t_{1}\right),\left(x_{2}, t_{2}\right) \in \Omega(T)$. But equations (7) are equivalent to the single equation (6). Therefore it must be shown that a Lipschitz function $u: \Omega(T) \rightarrow \mathbb{R}$ satisfying (6) also satisfies the integral equation (1).

We remark that the subsequent reasoning is based on the fact that a function belongs to the Sobolev space $W^{1, \infty}(\Omega(T))$ if and only if it admits a Lipschitz continuous representative. In particular, this representative is differentiable a.e. in $\Omega(T)$ and its derivatives are essentially bounded as elements of $L^{\infty}(\Omega(T))$ (see, e.g., [13]).

Define the set $\Theta(t, T):=\{(x, \tau): x \in[0, L],(\tau+x-t, \tau) \in \Omega(T)\}$, which depends on the value $T>0$ and the choice of $t \in[0, T]$. For each fixed $T>0$ and $t \in[0, T]$, suppose $\Psi(t, T): \Theta(t, T) \rightarrow \Omega(T)$ is a map such that $(x, \tau) \mapsto(\tau+x-t, \tau)$. Since $u$ belongs to the Sobolev space $W^{1, \infty}(\Omega(T))$ and the map $\Psi$ is invertible, with $\Psi$ and $\Psi^{-1}$ Lipschitz functions, we conclude that $u \circ \Psi \in W^{1, \infty}(\Theta(t, T))$, and, by the chain rule, we have

$$
\frac{d u(\tau+x-t, \tau)}{d \tau}=\frac{\partial}{\partial t} u(\tau+x-t, \tau)+\frac{\partial}{\partial x} u(\tau+x-t, \tau) \quad \text { for a.e. } \quad(x, \tau) \in \Theta(t, T)
$$

(see [13]). Therefore we may differentiate (6) with respect to $\tau$ to obtain (5), and then, using (18), we derive

$$
\frac{\partial}{\partial t} u(\tau+x-t, \tau)+\frac{\partial}{\partial x} u(\tau+x-t, \tau)=-m(\tau+x-t) u(\tau+x-t, \tau) \quad \text { for a.e. } \quad(x, \tau) \in \Theta(t, T),
$$

with any fixed $T>0$ and $t \in[0, T]$; or equivalently, we have

$$
\frac{\partial}{\partial t} u(x, t)+\frac{\partial}{\partial x} u(x, t)=-m(x) u(x, t) \text { for a.e. } \quad(x, t) \in \Omega .
$$

Further, because $u(\cdot, t) \in W^{1, \infty}(0, L)$ for all $t \in[0, T]$, it follows that the fundamental theorem of calculus is valid; that is, for all $\left(x_{1}, t\right),\left(x_{2}, t\right) \in \Omega(T)$,

$$
u\left(x_{2}, t\right)-u\left(x_{1}, t\right)=\int_{x_{1}}^{x_{2}} \frac{\partial}{\partial x} u(x, t) d x
$$

Similarly, we have

$$
u\left(x, t_{2}\right)-u\left(x, t_{1}\right)=\int_{t_{1}}^{t_{2}} \frac{\partial}{\partial t} u(x, t) d t
$$

for all $\left(x, t_{1}\right),\left(x, t_{2}\right) \in \Omega(T)$.

We now integrate equation (19) over $x_{1} \leq x \leq x_{2}, t_{1} \leq t \leq t_{2}$ to obtain

$$
\int_{x_{1}}^{x_{2}} \int_{t_{1}}^{t_{2}} \frac{\partial}{\partial t} u(x, t) d t d x+\int_{t_{1}}^{t_{2}} \int_{x_{1}}^{x_{2}} \frac{\partial}{\partial x} u(x, t) d x d t=-\int_{t_{1}}^{t_{2}} \int_{x_{1}}^{x_{2}} m(x) u(x, t) d x d t .
$$

Using relations (20), (21), we can easily rewrite the previous equation as the conservation law (1).

\section{Approximation of the solution}

The Banach fixed-point theorem, as well as its generalization used above, provide an approximation method for solving the system of integral equations (14) and therefore finding a solution of the predator-prey model. 
Precisely, for any nonnegative, continuous functions $B_{0}$ and $P_{0}$ that meet the requirements of the space $\mathscr{B}(T)$ (the pair $\left(B_{0}, P_{0}\right)$ is taken to be an initial approximation), the sequence of iterates $\left(B_{0}, P_{0}\right), \mathscr{M}\left(B_{0}, P_{0}\right), \mathscr{M}\left(\mathscr{M}\left(B_{0}, P_{0}\right)\right), \ldots$ converges in the uniform norm topology to the fixed point of the mapping $\mathscr{M}$. Thus we may define the iteration scheme for all $t \geq 0$ and nonnegative integers $n$ :

$$
\begin{aligned}
& B_{n+1}(t)=\left(\left(1-\varkappa P_{n}(t)\right)\left(\int_{0}^{t} b(x, t) B_{n}(t-x) \pi(x) d x+q(t)\right)\right)^{+}, \\
& P_{n+1}(t)=P_{0} \exp (-\mu t)+\beta \int_{0}^{t} P_{n}(\tau) \exp (-\mu(t-\tau))\left(\int_{0}^{\tau} b(x, \tau) B_{n}(\tau-x) \pi(x) d x+q(\tau)\right) d \tau .
\end{aligned}
$$

Proceeding in this manner, we generate the sequence $\left(B_{1}, P_{1}\right),\left(B_{2}, P_{2}\right),\left(B_{3}, P_{3}\right), \ldots$ of iterates, called the Picard iterates, that, under conditions of theorem 1 , converges to the solution of system (14) in the $\|\cdot\|_{\infty, T}$ norm for all $T>0$. Denoting this solution by $\left(B_{*}, P_{*}\right)$, we have $\left\|\left(B_{n}, P_{n}\right)-\left(B_{*}, P_{*}\right)\right\|_{\infty, T}$ approaches zero as $n \rightarrow+\infty$, and therefore $B_{n}$ and $P_{n}$ both converge uniformly to the limiting functions $B_{*}$ and $P_{*}$, respectively, on each interval $[0, T]$ for all $T>0$.

The sequence $\left(B_{n}, P_{n}\right)$ constructed above gives the sequence of approximate solutions $\left(u_{n}, P_{n}\right)$ of the predator-prey model, where the functions $u_{n}$ is determined explicitly by relations (16), where $u$ and $B$ must be replaced by $u_{n}$ and $B_{n}$, respectively; that is

$$
\begin{aligned}
& u_{n}(x, t)=u_{0}(x-t) \frac{\pi(x)}{\pi(x-t)}, \quad \text { for } \quad 0 \leq t \leq x, \\
& u_{n}(x, t)=B_{n}(t-x) \pi(x), \quad \text { for } \quad 0 \leq x \leq t .
\end{aligned}
$$

Let $\left(u_{*}, P_{*}\right)$ denote the exact weak solution of the predator-prey model. By the convergence of iterates, we conclude that $u_{n}$ converges uniformly to the limiting function $u_{*}$ on each set $\Omega(T)$ for all $T>0$.

To summarize, we have constructed the sequence of approximate solutions $\left(u_{n}, P_{n}\right)$ of the predator-prey model, and this sequence tends to the exact weak solution $\left(u_{*}, P_{*}\right)$ in the local uniform norm topology.

\section{Competing interests}

The authors declare that they have no competing interests.

\section{Authors' contributions}

All authors have contributed to all parts of the article. All authors read and approved the final manuscript.

\section{References}

[1] B. Charlesworth, Evolution in age-structured populations, Cambridge University Press, Cambridge, 1980.

[2] J. A. J. Metz and O. Diekmann, The dynamics of physiologically structured populations, Lect. Notes in Biomathematics, 68, Springer, Berlin-Heidelberg-New York, 1986.

[3] J. M. Cushing, An introduction to structured population dynamics, SIAM, Philadelphia, 1998.

[4] J. D. Murray, Mathematical biology I. An introduction, 3 rd ed., Springer, New York, 2002.

[5] J. M. Cushing and M. Saleem, A predator prey model with age structure, J. Math. Biology, 14 (1982), 231-250. 
[6] M. E. Gurtin and D. S. Levine, On predator-prey interactions with predation dependent on age of prey, Math. Biosciences, 47(3-4) (1979), 207-219.

[7] M. Saleem, Predator-prey relationships: egg-eating predators, Math. Biosciences, 65(2) (1983), 187-197.

[8] M. Saleem, Egg-eating age-structured predators in interaction with age-structured prey, Math. Biosciences, 70(1) (1984), 91-104.

[9] M. Saleem, S. U. Siddiqui, and V. Gupta, A mathematical model with young predation, J. of Math. Biology, 25(1) (1987), 89-101.

[10] M. Saleem and R. K. Pandey, Egg-eating age-structured predator and prey interactions: some simple cases, Math. Biosciences, 89(2) (1988), 209-224.

[11] J. D. Logan, An introduction to nonlinear partial differential equations, Wiley Interscience, New York, 2008.

[12] F. Brauer and C. Castillo-Chavez, Mathematical models in population biology and epidemiology, Springer, New York-DordrechtHeidelberg-London, 2012.

[13] G. Leoni, A first course in Sobolev spaces, AMS, Providence, 2009. 